\title{
Mutations in Caenorhabditis elegans Cytoplasmic Dynein Components Reveal Specificity of Neuronal Retrograde Cargo
}

\author{
Sandhya P. Koushika, ${ }^{1}$ Anneliese M. Schaefer, ${ }^{1}$ Rose Vincent, ${ }^{1}$ John H. Willis, ${ }^{2}$ Bruce Bowerman, ${ }^{2}$ and \\ Michael L. Nonet ${ }^{1}$ \\ ${ }^{1}$ Department of Anatomy and Neurobiology, Washington University School of Medicine, St. Louis, Missouri 63110, and ${ }^{2}$ Institute of Molecular Biology, \\ University of Oregon, Eugene, Oregon 97403
}

\begin{abstract}
We describe Caenorhabditis elegans dynein complex mutants, which misaccumulate synaptic proteins at the ends of neuronal processes. Ultrastructural analysis revealed irregularly sized vesicles that likely represent accumulation of cargo. We propose that synaptobrevin, synaptotagmin, and UNC-104 are specific cargoes of the dynein complex. Many cargoes link to dynein via interactions between dynactin and vesicle-associated spectrin. However, loss of spectrin results in only mild and occasional defects in synaptobrevin localization. Thus, the dynein-dynactin complex shows neuronal cargo selectivity without spectrin being a critical component of cargo binding. We observed parallels to progressive motor neuron disease symptoms in these animals. With age, neuronal misaccumulations increase in size and frequency; locomotion becomes progressively slower; and life span is shortened. These mutants provide a model to assess whether defects in transport of specific cargo mediate neuronal dysfunction.
\end{abstract}

Key words: retrograde; synapse; axonal transport (axoplasmic transport); dynein; dynactin; spectrin

\section{Introduction}

Transport of proteins to and from the synapse is essential for synaptic development and maintenance. The microtubule motor dynein is a key retrograde transporter of cargo from the synapse to the cell body (Goldstein and Yang, 2000; King et al., 2002). The dynein motor complex consists of two heavy-chain motor units, several light chains, intermediate chains, and light intermediate chains (King, 2000). Because the dynein motor complex transports several cargoes, it has been suggested that association with different accessory subunits can give rise to cargo specificity (Susalka et al., 2000). One such complex, dynactin, is intimately associated with many of the roles of dynein and links to the dynein complex through the intermediate chain (Waterman-Storer et al., 1997; King, 2000). The dynein-dynactin complex binds to transport vesicles through interaction with vesicle-associated spectrin and acidic phospholipids (Holleran et al., 1996, 2001; Muresan et al., 2001).

Genetic and antibody $(\mathrm{Ab})$ block approaches have demonstrated a role for dynein in axonal transport. Dynein heavy chain,

\section{Received Nov. 12, 2003; revised March 5, 2004; accepted March 8, 2004}

We thank Alex van der Bliek, Marty Chalfie, Lihisha Chen, Vann Bennet, Pierre Gronczy, and Maurine Linder for generously providing us with antibodies; the Caenorhabditis Genetics Center, Yishi Jin, and Eric Jorgensen for strains; John Yoder and Min Han for dli-1 plasmid DNA; Danielle Hammil, Diane Schmidt, and Susan Strome for sharing information about $d h c-1$ before publication; Grady Phillips for cutting electron microscopic sections; David Hall for help in interpreting electron microscopic data; and Krishanu Ray for critically reading this manuscript.

Correspondence should be addressed to Michael L. Nonet, Department of Anatomy and Neurobiology, Washington University School of Medicine, 660 South Euclid Avenue, St. Louis, M0 63110. E-mail: nonetm@wustl.edu, sandhya@pcg.wustl.edu.

DOI:10.1523/JNEUROSCI.5039-03.2004

Copyright $\odot 2004$ Society for Neuroscience $\quad$ 0270-6474/04/243907-10\$15.00/0 light chains, and a component of the dynactin complex are involved in organelle motility in the squid axoplasm (Schnapp and Reese, 1989; Hirokawa et al., 1990; Steffen et al., 1997; Waterman-Storer et al., 1997). Overexpression of dynamitin, which disrupts the dynein-dynactin interaction, causes defects in retrograde transport and mimics symptoms seen in amyotrophic lateral sclerosis (ALS) patients (LaMonte et al., 2002). Similarly, a human motor neuron disease is linked to a mutation in the p150 ${ }^{\text {Glued }}$ component of the dynactin complex (Puls et al., 2003). Drosophila mutations in the cytoplasmic dynein heavy chain, p150 Glued component of dynactin, and roadblock light chain all have transport defects wherein synaptic vesicle components and endosomal cargo clog the motor neuron axons (Bowman et al., 1999; Martin et al., 1999). Interestingly, some of these phenotypes are very similar to mutants in the anterograde kinesin pathways (Hurd and Saxton, 1996; Gindhart et al., 1998, 2003). The complexity of phenotypes in many systems and the requirement for cell survival (Harada et al., 1998) have made it difficult to identify components required for the retrograde transport of a single or subset of neuronal cargoes.

To better understand dynein-dependent transport, one needs to identify the many cargoes of dynein as well as the specific composition of the transporting complex. We used a synaptically localized protein, synaptobrevin, as a reporter of axonal transport and isolated mutants in the Caenorhabditis elegans cytoplasmic dynein heavy chain and dynein light intermediate chain genes (Schaefer et al., 2000). These mutants misaccumulate synaptobrevin at the ends of the neuronal process and are defective in trafficking a subset of synaptic proteins. The same proteins mis- 
accumulate when we disrupt the dynactin complex either by using a mutant in $\mathrm{p} 150$ Glued or by overexpressing dynamitin. Spectrin may not be essential to link motor and cargo because very mild phenotypes are seen in the null alleles of both $C$. elegans $\beta$-spectrin genes. Cellular and organismic phenotypes that parallel those seen in neurodegenerative diseases were also observed in the dynein complex mutants.

\section{Materials and Methods Immunocytochemistry}

Animals were fixed and prepared for immunocytochemistry using a modified Bouin's fixative or 2\% paraformaldehyde (Nonet et al., 1993, 1997). The following antibodies were used: mouse anti-RAB-3 (Nonet et al., 1997), rabbit anti-synaptotagmin (SNT-1; Nonet et al., 1993), mouse anti-SNT-1, rabbit anti-synaptobrevin (SNB-1; Nonet et al., 1998), chicken anti-synaptogyrin (SNG-1; Zhao and Nonet, 2001), rabbit antiMEC-7 (Savage et al., 1994), mouse anti-acetylated tubulin (Sigma, St. Louis, MO; Fukushige et al., 1999), mouse anti-UNC-104 (S. P. Koushika and M. L. Nonet, unpublished data), anti-UNC-70 (Moorthy et al., 2000), rabbit anti-DYN-1 (Labrousse et al., 1998), rabbit anti-Rim (Koushika et al., 2001), mouse anti-green fluorescent protein (GFP; Clonetech, Cambridge, UK; or Roche Molecular Biochemicals, Indianapolis, IN), rabbit anti-GFP (a gift from M. Linder, Washington University), rabbit anti-UNC-64 (Saifee et al., 1998), and rabbit anti-DHC-1 (Gonczy et al., 1999). Appropriate secondary Alexa 488 and Alexa 568 antibodies were used (Molecular Probes, Eugene, OR). Images were captured using MTI-SIT68 or Retiga EXi-cooled CCD cameras with Scion Image and processed with Adobe Photoshop and Canvas.

\section{Electron microscopy}

Protocols have been described by Koushika et al. (2001). The position and the presence of distinctive large-diameter microtubules identified ALM processes. The developed negatives were digitized using a Canon EOS-D30 digital camera.

\section{Genetic analysis}

All strains were maintained as described by Sulston and Hodgkin (1988). Colchicine (Sigma) was added as a solid into partially cooled agar and stirred until dissolved. Two ${ }_{\mathrm{p}}$ mec-7::SNB-1::GFP integrated transgenes were used, jsIs37V and jsIs40I; both had identical patterns of GFP fluorescence. $j u I s 1 \mathrm{X}$ is an integrated transgene, $\mathrm{p}_{\mathrm{p}} u$ - $15:: \mathrm{SNB}-1:: \mathrm{GFP}$, expressed in GABAergic VD and DD motor neurons (Hallam and Jin, 1998). Three-factor mapping with $d p y$-5(e61) unc-13(e51)I, unc-11(e47) $d p y-5(e 61) \mathrm{I}$, and $u n c-74(x 19) d p y-5(e 61) \mathrm{I}$ was performed. Six of six Unc-13 non-Dpy-5 carried js121, whereas zero of four Dpy-5 nonUnc- 13 carried $j$ s121. Sixteen of 24 Dpy- 5 non-Unc- 11 carried js 121 , and 4 of 10 Unc-11 non-Dpy-5 carried js121. js319 also mapped between unc-11 and $d p y-5$. Twelve of 12 Dpy-5 non-Unc-74 and 0/5 Unc-74 non-Dpy-5 carried js121. js121 was further mapped using singlenucleotide polymorphisms (SNPs). js351 was mapped using $d p y$ 13(e458) unc-5(e53)IV. Thirteen of 13 Dpy-13 non-Unc-5 recombinants carried js351. js351 was uncovered by the deficiency $s D f 22$ that maps to the right of unc-5. or404ts was mapped using unc-8(e49)dpy20(e1282)IV. Twenty-two of 28 Unc-8 non-Dpy-20 carried or 404ts, whereas 2 of 20 Dpy-20 non-Unc- 8 carried or 404 ts. or 404 ts embryos also had a mitotic spindle defect similar to those seen using $d n c-1$ RNAi (Skop and White, 1998).

\section{PCR mapping}

Chromosome assignments were made using the Bergerac strain (Williams et al., 1992). js121 was SNP-mapped because of its strong penetrance and sterility. Sterility and SNB-1 misaccumulations were assumed to arise from the same locus. unc-11(e47) js121 dpy-5(e61)/unc-74(x19) $d p y$-5(e61); jsIs37 was crossed to CB4856 males. Sixty-three Dpy-5 nonsteriles and 10 Unc-11 nonsteriles were assessed for the presence of the following SNPs: Y119C1B at position 23692, C09D1 at position 24285, T03A1 at position 28270, and C41D11 at position 26816 (Wicks et al., 2001). Seven Dpy-5 nonsteriles and one Unc-11 nonsterile placed js121 between C41D11 and T03A1, a fivecosmid interval. Eight unc-11(e47) js121; jsIs37 and five js121 dpy-5(e61);
Table 1. Area of SNB-1::GFP misaccumulations

\begin{tabular}{lll}
\hline & $\begin{array}{l}\text { Animals with } \\
\text { misaccumulations } \\
\text { of SNB-1::GFP }\end{array}$ & $\begin{array}{l}\text { Size of SNB-1::GFP } \\
\text { misaccumulations } \\
\left(\mu \mathrm{m}^{2}\right)(\text { SEM) }\end{array}$ \\
\hline js/s37 (wild type) & $0 / 40$ & \\
dhc-1(js121) & $20 / 20$ & $4.74(0.62)^{*}$ \\
sma-1(ru18) & $13 / 40$ & $1.88(0.22)^{*}$ \\
unc-70(e524) & $3 / 48$ & $N D$ \\
unc-70(s1502) & $5 / 20$ & $0.31(0.08)^{*}$ \\
dnc-2 overexpression & $20 / 20$ & $5.04(0.4)$
\end{tabular}

Misaccumulations in various mutant backgrounds are shown. An animal with any misaccumulation in any of the three neuronal processes that ended in the nose was counted as positive. To determine size of accumulations, all animals were imaged with a $C \mathrm{CD}$ camera at the same gain, light level, and magnification. Area measurements were made using Image J. ND, Not determined.

*dhc-1(js121);js/s37 is significantly different from sma-1(ru18):js/s37 ( $\approx 2.5$ times less) and unc-70(1502);js/s37 at $p<2 \times 10^{-4}$

jsIs37 were also selected; however, because they were sterile, the DNA extracted was useful only for four or five PCR reactions. One js121 dpy-5(e61) and two unc-11(e47) js121 placed js121 in the interval between T03A1 and Y119C1B, an eight-cosmid interval consistent with the data obtained using fertile non-js121 recombinants.

\section{Plasmid constructions}

The $d h c-1$ full-ength clone (NM1035) was built by subcloning and sequencing a $3.3 \mathrm{~kb}$ PstI-SalI PCR fragment $1.7 \mathrm{~kb}$ promoter region and nearly three N-terminal exons into Bluescript (Stratagene, La Jolla, CA). Subsequently a DraII-KpnI $16.7 \mathrm{~kb}$ fragment from the cosmid T21E12 containing the remainder of the gene was inserted into identical sites of the above clone (NM1035). $d n c-2$ was PCR-amplified from genomic DNA and was cloned into ${ }_{p} m e c-7$ vector pPD96.41 (NM1170).

\section{Transformation rescue}

dli-1. pJHY10, a StuI-XhoI fragment of dli- 1 containing $3.5 \mathrm{~kb}$ upstream, $1.6 \mathrm{~kb}$ genomic open reading frame, and $450 \mathrm{bp}$ of the $3^{\prime}$ untranslated region, was used for rescue (Yoder and Han, 2001). pJHY10 at $15 \mathrm{ng} / \mu \mathrm{l}$ and pRF4 at $150 \mathrm{ng} / \mu \mathrm{l}$ were injected into $d l i-1(j s 351) / d p y-20(e 1282) u n c-$ 31(e169) unc-26(e205); jsIs37. js351 animals segregating from the lines were scored for sterility and misaccumulation defects. The severity of the phenotype was scored from 0 (no phenotype) to 3 (large accumulation) in 4 - to 6-d-old animals. js351 from the uninjected strain: 0,0 of $38 ; 1,2$ of $38 ; 2,26$ of 38 ; and 3,10 of 38 . Line 5 rollers: 0,14 of $23 ; 1,4$ of $23 ; 2$, 4 of 23 ; and 3, 1 of 23 . Line 6 rollers: 0,18 of $38 ; 1,10$ of $38 ; 2,10$ of 38 ; and 3,0 of 38 . Fourteen animals in line 6 and seven animals in line 5 had modest fertility and threw roller and nonrollers with and without the GFP phenotype, respectively. There were no fertile $j s 351$ animals from the parent strain.

dhc-1. NM1035 was injected into $d h c-1(j s 319) ; j s I s 37$ at $3 \mathrm{ng} / \mu \mathrm{l}$ with pRF4 at $150 \mathrm{ng} / \mu \mathrm{l}$. The severity of the phenotype was scored from 0 (no phenotype) to 3 (large accumulation) in 2- to 4-d-old animals. js319 from uninjected strain: 0,1 of $22 ; 1,5$ of $22 ; 2,13$ of 22 ; and 3,3 of 22 . Line 1 rollers: 0,12 of $20 ; 1,5$ of $20 ; 2,3$ of 20 ; and 3,0 of 20 . Line 2 roller animals: 0,17 of $24 ; 1,3$ of $24 ; 2,4$ of 24 ; and 3, 0 of 24 . The same arrays did not alter $j$ sIs 37 SNB-1::GFP localization.

$d n c-1$ (or404ts). Cosmid ZK593 containing the $d n c-1$ gene was injected into or 404 ts at $5 \mathrm{ng} / \mu \mathrm{l}$ with pRF4 at $50 \mathrm{ng} / \mathrm{ul}$. Genomic yeast DNA was used as a carrier at a concentration of $50 \mathrm{ng} / \mu \mathrm{l}$. Three lines were obtained in which the embryonic lethality and mitotic spindle orientation defects of or $404 t s$ were rescued at the restrictive temperature in animals that grew up to be positive for pRF4.

\section{Misaccumulation size measurement}

Animals were picked as L4 larvae and aged either 1 or $5 \mathrm{~d}$. They were imaged at the same time using an identical intensity of light and gain on the camera. Area was measured using Image J. The area measurements of other mutants for Table 1 were done using randomly picked animals from a growing plate. Significance was calculated using pair-wise one-tail Student's $t$ test. 

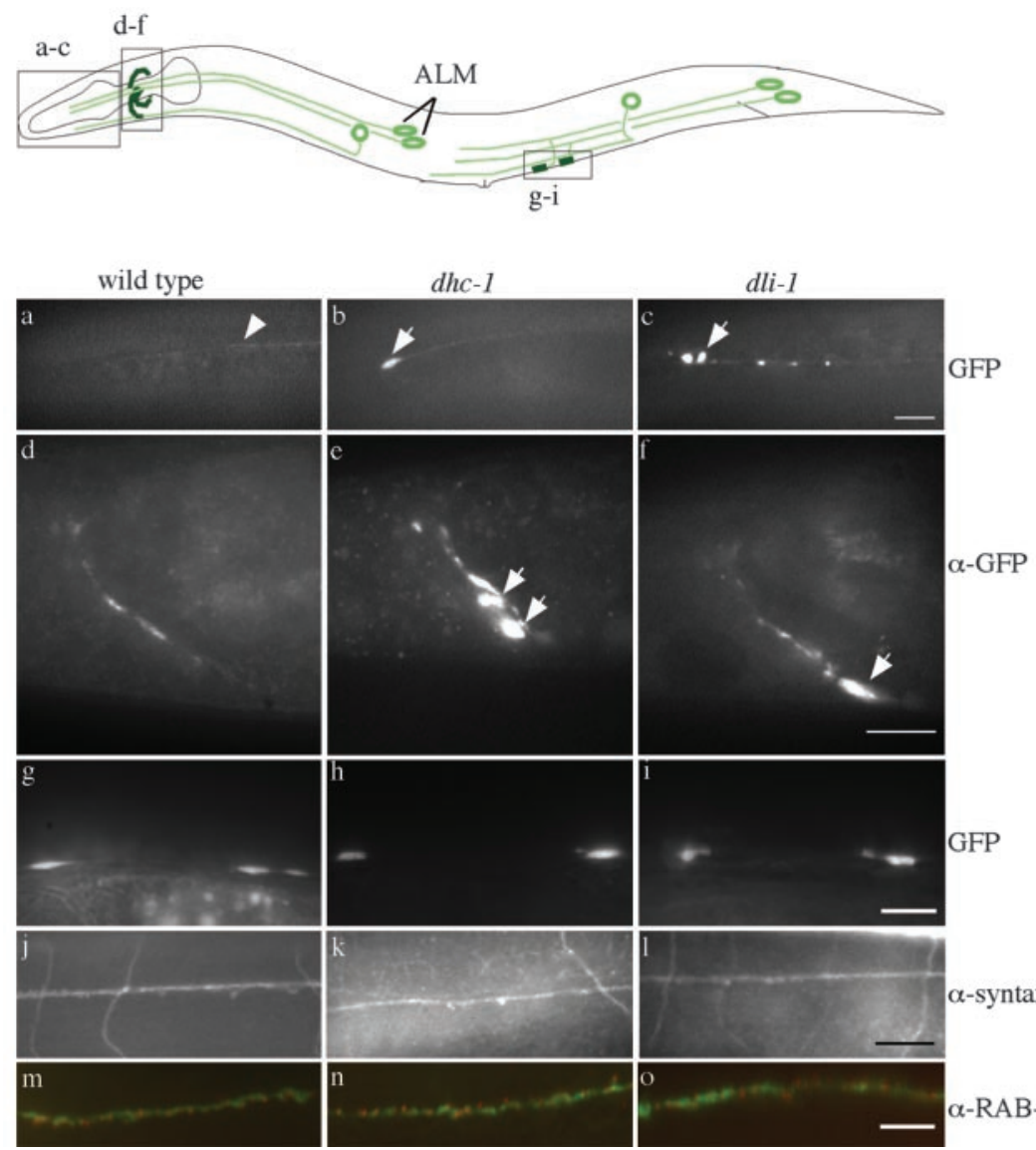

Figure 1. Neuronal architecture of misaccumulation mutants. Top, Schematic diagram of worms expressing synaptobrevin::GFP in mechanosensory neurons. ALMs are a pair of lateral mechanosensory cells that extend neuronal processes into the nose and nerve ring. $a-c$, js/s37 ( $a$ ), dhc-1(js319); js/s37(b), and dli-1(js351); js/s37(c) noses; $b$ has one misaccumulation, whereas the neuronal process in c has multiple misaccumulations. The arrowhead in $a$ shows the main ALM neuron process, and arrows in $b$ and c show misaccumulations of SNB-1::GFP near the tip of ALM processes. $d-f, j s / s 37, d h c-1(j s 121) ; j s / s 37$, and $d l i-1(j 5351) ; j s / s 37$ nerve ring area. Mutants have hyperaccumulations of synaptobrevin expression indicated by the arrows. $g-i$, js/s37, dhc-1(js319); js/s37, and dli-1(js351); js/s37 patches in the ventral cord, which appear grossly similar. j-l, js/s37, dhc1(js121); js/s37, and dli-1(js351); js/s37 immunostaining for syntaxin, which marks all neuronal processes. Seen here is an image of the dorsal cord and neuronal commissures; the mutants appear similar to wild type. $m-0, j s / s 37, d h c-1(j s 121)$; js/s37, and dli-1(js351); js/s37 immunostained for RAB-3 (green) and Rim (red) from the ventral cord. Mutant animals are indistinguishable from wild type. Scale bars: $10 \mu \mathrm{m} ; 0,5 \mu \mathrm{m}$.

\section{Velocity measurements}

Measurements were done as described previously with images being captured every $1 \mathrm{sec}$ (Staunton et al., 2001). Significance was calculated using pair-wise one-tail Student's $t$ test.

\section{Life span}

Animals were collected as L4s and moved every $2 \mathrm{~d}$ to a fresh plate until the end of their reproductive life, after which they remained on the same plate. Animals were scored as dead if they did not move when prodded with a platinum pick and did not show pharyngeal pumping. Significance was calculated using pair-wise one-tail Student's $t$ test.

\section{Results}

\section{Four mutants that misaccumulate synaptobrevin::GFP}

We performed a genetic screen to isolate putative transportdefective mutants using tagged synaptic vesicles. Synaptic vesicles were visualized in the six $C$. elegans mechanosensory neurons using a stable line, jsIs37, which expresses GFP-tagged SNB-1 (SNB-1::GFP). These neurons have one major neuronal process extending anterior from the cell body (Chalfie, 1993). Five of six neuronal processes extend a branch into the nerve ring or ventral cord, whereas the primary process continues anteriorly. Synaptic
$\alpha-G F P$

vesicle proteins such as synaptobrevin are enriched at synapses along the neuronal branch (Fig. 1d,g). In jsIs37 (wild-type), animals the GFP fluorescence is punctate and primarily concentrated at synaptic regions in the nerve ring and ventral cord (Fig. 1, schematic) (Nonet, 1999).

A genetic screen in which 2032 haploid genomes were screened using jsIs37 was performed (Schaefer et al., 2000). In this screen, four putative transport-defective mutants ( $j$ s319, js121, js320, and js351) in three complementation groups that misaccumulate SNB-1::GFP were isolated. The misaccumulations and hyperaccumulations occur near the tips of the mechanosensory neuronal processes (Fig. $1 a-c$ ) and near nerve ring synapses (Fig. $1 d-f$ ), respectively. In these mutants, the synapses along the ventral cord appeared grossly normal (Fig. $1 g-i$ ). All mutants isolated had superficially normal patterns of outgrowth and connectivity, as observed by anti-syntaxin (Fig. $1 j-l$ ), anti-Rim, and anti-RAB-3 immunocytochemistry (Fig. $1 m-o)$. Western analysis documented that the overall protein levels of synaptotagmin, syntaxin, and synaptobrevin in js319 were indistinguishable from those of wild type (data not shown). In 1- to 2-d-old adult animals, the ultrastructure of neuromuscular junctions and the organization of the large-diameter mechanosensory microtubules of $j s 121$ and js351 were indistinguishable from those of wild type (Fig. $2 a-g$ ). Although $C$. elegans SAB motor neurons sprout in response to absence of an unknown signal from the postsynaptic muscle cell (Zhao and Nonet, 2000), no such sprouting is seen in either js121 or js351 mutant animals (data not shown). Furthermore, in young adults, no defects were seen in an aldicarb assay that assesses cholinergic neuromuscular transmission (data not shown). These data suggest that the mutated genes were not required for neuronal or synaptic development and instead had defects in localizing synaptobrevin.

The mutants also had non-neuronal phenotypes. Three mutants, js351, js121, and js319, have a mildly dumpy body; both $j s 351$ and js121 have protruding vulva and are sterile, and js351 sometimes develops blisters and bumps on its cuticle. js319 also segregates $1-2 \%$ of males in its progeny, indicative of nondisjunction during chromosome segregation. The non-neuronal phenotypes suggest roles for these genes outside the nervous system.

\section{The mutants encode components of the cytoplasmic} dynein complex

To determine the molecular identity of the mutants, two complementation groups were mapped and cloned (for details, see Materials and Methods). js351 was mapped to the right arm of chromosome IV to a region that contained the candidate gene dynein light intermediate chain, $d l i-1$. The identity of $j s 351$ as $d l i-1$ was confirmed by several criteria. A previously identified allele, dli- 
1(ku266), did not complement js351 and had identical misaccumulation phenotypes (Yoder and Han, 2001) (data not shown). Sequencing the $d l i-1$ gene in $j s 351$ identified a stop codon (CAA to TAA) at amino acid $\mathrm{Gln}^{218}$. We rescued the misaccumulation phenotype using a transgene containing the genomic region of dli-1. Thus, we identified js351 as a null mutation in C. elegans dli-1. js121 and js319 belong to a single complementation group; js121 was mapped to a region containing five cosmids. One candidate gene in this interval was the cytoplasmic dynein heavy chain, dhc-1 (Gonczy et al., 1999). A transgene containing the genomic region of dhc- 1 rescued the misaccumulation phenotypes of js319. Both our alleles, js319 and js121, also did not complement another known allele, dhc-1(or195), which had identical misaccumulation phenotypes
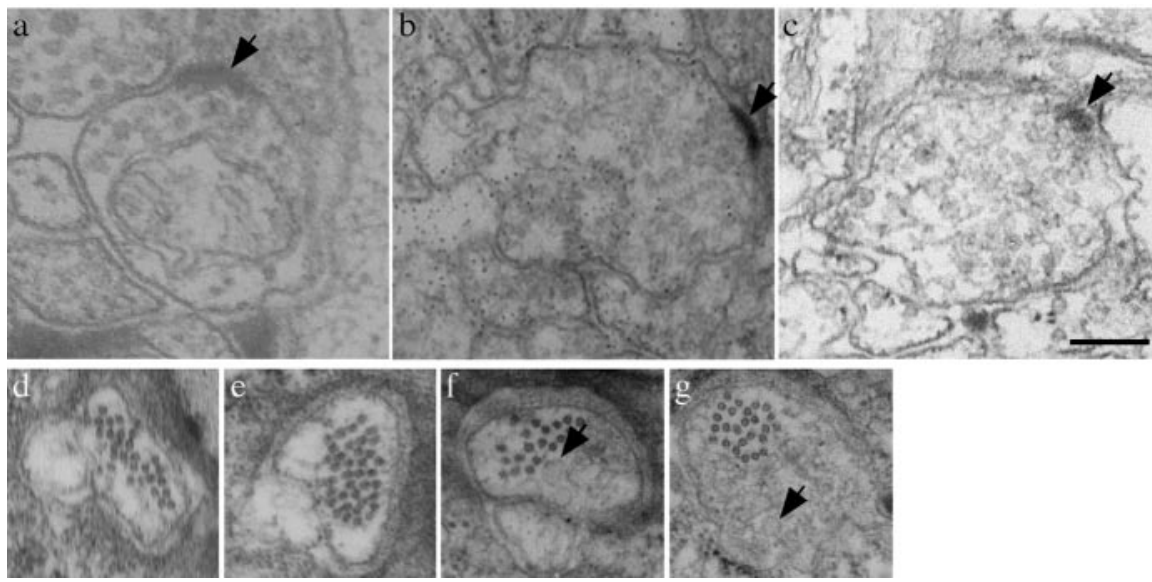

Figure 2. Ultrastructure of $d h c-1$ and dli-1. $a-c$, Ventral cord neuromuscular junction electron micrographs of 1-to 2-d-old adult animals: wild type (a), dhc-1(js121); js/s37 (b), and dli-1(js351); js/s37 (c). The arrow points to the active zone, and the profile shows the presence of synaptic vesicles. $d-g$, Electron micrographs of ALM neuron process showing the large-diameter microtubules in wild type ( $d$ ) and $d h c-1$ (js 121); js/s37 (e) 1- to 2-d-old adults. $f, g$, Collection of vesicles (arrows) in dhc-1(js 121); js/s37 ALM process in 1- to 2-d-old adult animals. Scale bar, $200 \mathrm{~nm}$.
(Hamill et al., 2002). Thus, we identified

js121 and js319 as alleles of $d h c-1$. The mutant phenotypes attest to widespread roles in cellular function. Other non-neuronal phenotypes of $d h c-1$ and $d l i-1$ mutants have been described elsewhere (Gonczy et al., 1999; Yoder and Han, 2001). In this study, we concentrate on neuronal phenotypes of the dynein complex mutants. Thus, our screen was successful in identifying core components involved in axonal transport.

\section{Misaccumulations are microtubule-dependent}

Dynein is a microtubule motor; therefore, we wanted to ensure that the misaccumulations do not arise by microtubuleindependent processes such as aberrant protein sorting. Mechanosensory neurons have unique large-diameter 15-protofilament microtubules, which are made of cell-specific $\alpha$-MEC-12 and $\beta$-MEC-7 tubulins (Savage et al., 1989; Fukushige et al., 1999). Worms were grown in the microtubule-depolymerizing drug colchicine, which greatly reduces the microtubule cytoskeleton of only mechanosensory neurons without affecting their neuronal process extension (Chalfie and Thomson, 1982). Wild-type worms grown on $0.8 \mathrm{~mm}$ colchicine resulted in absence of GFP in synaptic regions and the nose (Fig. 3c), with most of the fluorescence concentrated in the cell body and proximal part of the neuronal process (data not shown). $d h c-1$ and $d l i-1$ animals grown on colchicine did not have any misaccumulations at the end of the neuronal process, but variable nerve ring staining was still visible (Fig. $3 d, e$ ). This may be attributable to the residual microtubules remaining after colchicine treatment at this concentration. A similar but less dramatic reduction of the misaccumulations was seen in double mutants between dynein complex components and the cell-specific tubulins (data not shown). These data show that the presence of misaccumulations requires an intact microtubule cytoskeleton.

\section{The misaccumulations arise because of retrograde defects}

To test the dependence of the misaccumulations on anterograde transport, double mutants were made with the dynein complex mutants and the anterograde kinesin family motor UNC-104, which transports synaptic vesicles in neurons (Hall and Hedgecock, 1991; Otsuka et al., 1991). In unc-104(e1265), a strong hypomorph, SNB-1::GFP is primarily present in the cell body, and little or no fluorescence is seen in synaptic regions (Nonet, 1999). In double mutants with 1265 and $d h c-1(j s 319)$, the misaccumu-
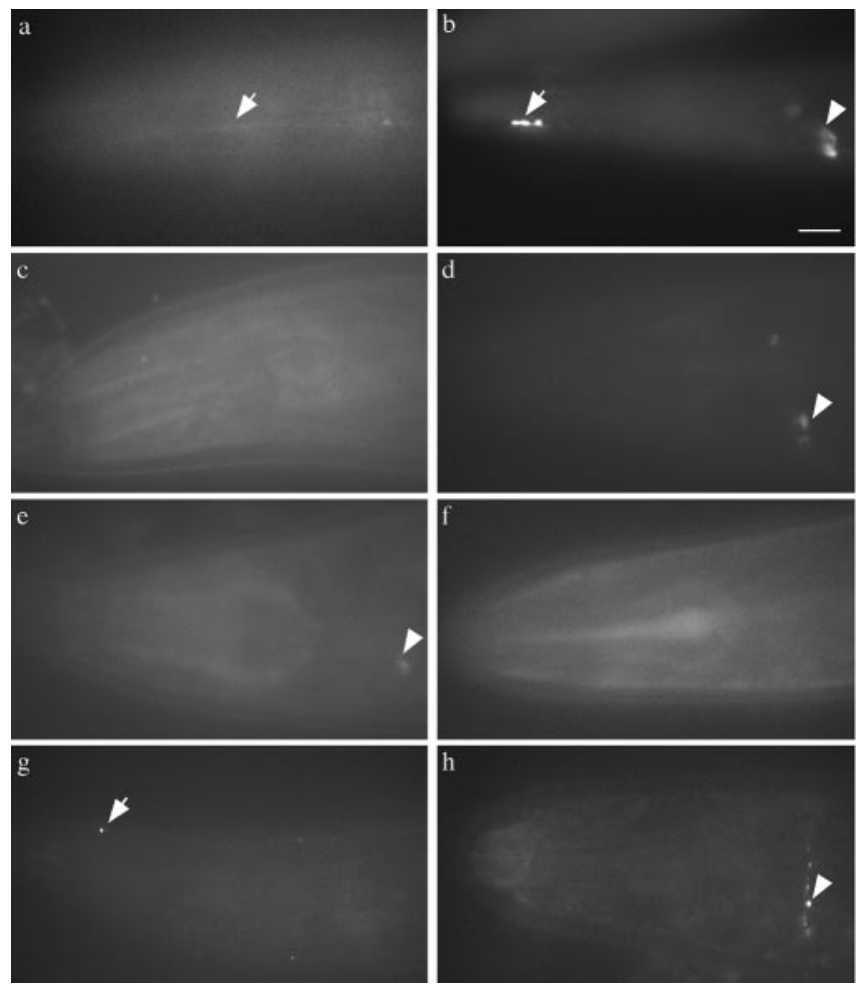

Figure 3. Misaccumulations in the nose are dependent on a kinesin family motor and microtubules. $a$, The arrow points to the ALM process in $j / 537 . b$, The arrow points to a misaccumulation at the tip of the ALM process in dhc-1(js121); js/s37. (-e, Phenotype of worms grown in $0.8 \mathrm{~mm}$ colchicine. $c, j / 537$, no signal in nose or nerve ring. $d$, dhc-1(js 121);j/s37, some signal in the nerve ring (arrowhead) but no misaccumulation of SNB-1::GFP in the nose. e, dli-1(j3351);js/s37, some signal in the nerve ring (arrowhead) but SNB-1::GFP misaccumulation in the nose is absent. $f-h$, unc-104 kinesin-like protein double mutants. $f$, unc-104(e1265); is/s37 animals have no signal in the nose. $g$, dhc-1(js121); unc-104(e1265); jsls37. Some animals have misaccumulations (arrow), but they are significantly smaller (compare with b). h, unc-104(e1265); dli-1(j3351); ;sls37. Misaccumulations are absent, although some GFP is present in the nerve ring (arrowhead). Scale bar, $10 \mu \mathrm{m}$.

lations are either completely absent $(80 \%)$ or highly reduced compared with $d h c-1(j s 319)$ alone. Similarly, in $d h c-1(j s 121)$; unc-104(e1265) and unc-104(e1265); dli-1(js351) animals, the misaccumulations are either reduced (90\%; Fig. 3, compare $g, b$ ) 

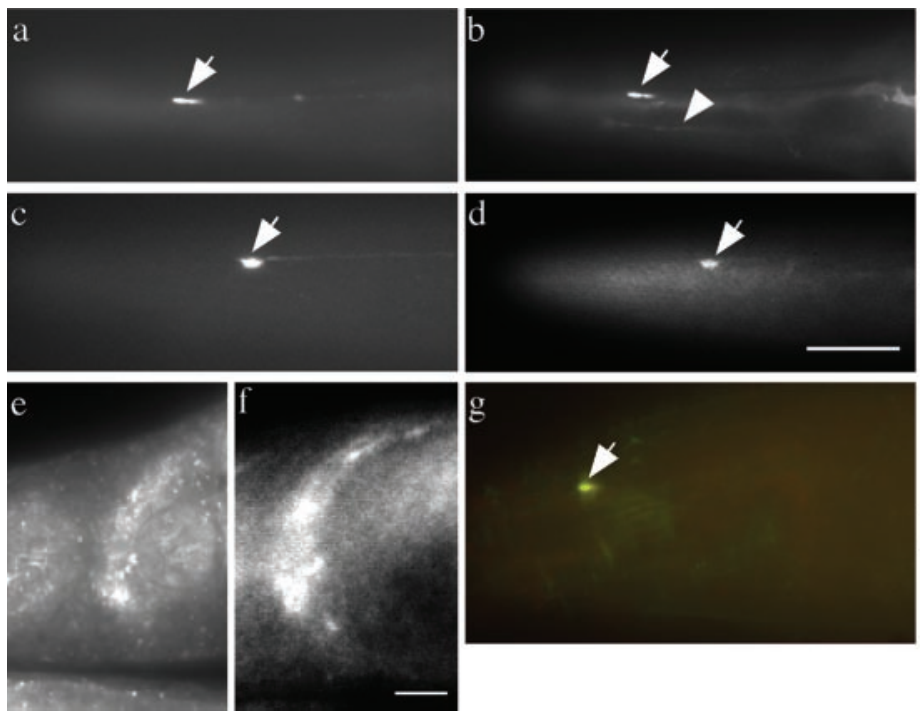

Figure 4. Dynein complex mutants misaccumulate the anterograde kinesin family motor UNC-104 and native synaptobrevin. $a-d$, GFP (first image of set) and UNC-104 (second image of set) Ab staining. $a$, $b$, dhc-1(js319); js/s37 SNB-1::GFP misaccumulations (arrow) in the nose also contain the UNC-104 protein. The arrowhead in $b$ points to UNC-104 protein in a SAB neuron. $c, d$, dli-1(j3351); js/s37 SNB-1::GFP misaccumulations (arrow) in the nose also contain UNC-104 protein. $e$, $f$, Dynein heavy chain immunostaining. e, Nerve ring staining in wild type showing that the heavy chain is enriched in neurons. $f$, Immunoreactivity in unc-104(e1265) animals still shows DHC-1 protein enriched in the nerve ring. $g$, Double labeling for synaptotagmin (green) and synaptobrevin (red) staining in $d h c-1(j 3319)$ animals lacking js/s37 showing a misaccumulation containing both proteins (arrow). Scale bars, $10 \mu \mathrm{m}$.

or absent (10\%; Fig. 3h). Because the SNB-1::GFP misaccumulations in the mutants are still UNC-104-dependent, this suggests that vesicles containing synaptobrevin in the mutants still retain characteristics of synaptic vesicles. Thus, in these mutants, sorting of synaptobrevin is likely to be unaffected, and the primary defect is the altered transport of vesicles containing synaptobrevin. In addition, anterograde transport must be functional for the dynein-dependent phenotypes to be seen.

To determine whether the phenotypes can arise from retrograde defects, we looked at the accumulation of the anterograde motor UNC-104, which can return to the cell body only by means of functional retrograde transport. Double immunolabeling with both anti-UNC-104 and anti-GFP showed that UNC-104 does accumulate with SNB-1::GFP (Fig. $4 a-d$ ), demonstrating that at least some UNC-104 cannot return to the cell body. However, DHC- 1 that is present in the nerve cords and nerve ring itself is independent of UNC-104 for its transport (Fig. 4e,f). The simplest interpretation consistent with these observations is that the dynein complex mutants alter retrograde axonal transport.

Misaccumulations are vesicles containing synaptobrevin and synaptotagmin but not other presynaptic proteins

Because dynein is a major retrograde motor, one can expect that it transports many cargoes including other synaptically localized proteins. To determine whether native synaptobrevin behaved similarly to the SNB-1::GFP fusion protein, mutant animals lacking the transgene were double-stained for SNT-1 and SNB-1. Endogenous SNB-1 accumulated in regions where SNT-1 (see below) accumulated (Fig. $4 g$ ). To identify other cargo, we performed immunostaining analyses for various neuronal proteins that misaccumulate along with synaptobrevin. Animals carrying jsIs37 in the mutant background were immunolabeled for GFP and the synaptic vesicle markers synaptotagmin, RAB-3, and SNG-1. The GFP misaccumulations at the ends of the neuronal processes contain synaptotagmin (Fig. $5 a-f$ ) but not RAB-3 (Fig. dhc-1

GFP/UNC-104

dli-1

GFP/UNC-104

dhc-1

SNB-1/SNT-1

$5 g-i$ ) or synaptogyrin (Fig. $5 j-l$ ). The misaccumulations also failed to stain for either dynamin or Rim, both of which are presynaptic markers not present on synaptic vesicles (data not shown) (Labrousse et al., 1998; Koushika et al., 2001). The other domains of expression of these vesicular and presynaptic components appear grossly normal (see above; Figs. $4 b, 5 f, l$, $\mathrm{SAB}$ neuron). These data suggest that sites of SNB-1::GFP misaccumulations also contain synaptotagmin but are not likely to be ectopic synapses because many proteins known to be present at synapses, such as, Rim are absent. UNC-64 syntaxin, a transmembrane protein that is expressed on the plasma membrane of neurons, also misaccumulates occasionally (40-50\%; Fig. 5m-r) (Saifee et al., 1998). Taken together, the simplest explanation is that the defects arise because of inappropriate transport of vesicles containing synaptobrevin, synaptotagmin, and UNC-104 but lacking other presynaptic components. Furthermore, the dynein complex is likely to transport a portion of syntaxin, and the rest may be trafficked by other motors.

To determine the nature of these misaccumulations, an ultrastructural analysis of the mechanosensory neurons in 1- to 2-d-old adult animals was performed. Near the tips and occasionally along the neuronal process, variably sized irregularly shaped vesicle profiles were seen (Fig. $2 f, g$ ) which were absent in wild-type mechanosensory neurons (Fig. $2 d$ ). The most straightforward explanation is that cargoes such as synaptobrevin are present on these irregular vesicles, which are retrogradely transported by the dynein complex.

\section{Disrupting dynactin results in the same spectrum of cargo}

The dynein motor complex often works in close association with the multiprotein dynactin complex. To test the role of this complex, we used a partial loss-of-function allele, $d n c-1$ (or404ts), in its largest subunit, p150 Glued (see Materials and Methods). This allele carried a missense mutation $\mathrm{R}$ to $\mathrm{C}$ at position 1237 (CGT to TGT), which is near the region that binds the actin-related protein-1 (ARP1) filament of dynactin (Waterman-Storer et al., 1995). We also overexpressed $d n c-2$ dynamitin (Skop and White, 1998), which dissociates the dynactin complex (Echeverri et al., 1996), in the mechanosensory neurons. Both these manipulations resulted in misaccumulations of GFP-tagged synaptobrevin at the tips of the neuronal processes and hyperaccumulations in the region of the nerve ring (data not shown). Immunostaining revealed that the SNB-1::GFP misaccumulations contain synaptotagmin (Fig. 6a-f), UNC-104 (Fig. $6 g-l$ ), and occasionally syntaxin (Fig. $6 m-r$ ) but do not contain other proteins that were unaffected in the dynein complex mutants (data not shown). This demonstrates that the dynactin complex is required for the transport of vesicles containing synaptobrevin, synaptotagmin, and UNC-104.

\section{Spectrin mutants do not alter transport of synaptobrevin} Vesicle-associated spectrin has been shown to be important for attaching the dynein-dynactin complex to its cargo and supporting its motility (Holleran et al., 1996, 2001; Muresan et al., 2001). 
We wanted to determine whether spectrin was required for transporting synaptic vesicle proteins. A functional spectrin cytoskeleton comprises heterotetramers of two $\alpha$ and two $\beta$ subunits (Bennett and Baines, 2001). C. elegans has only three genes that encode the $\alpha$ - and $\beta$-spectrins, namely spc-1, unc-70, and sma-1 (McKeown et al., 1998; Hammarlund et al., 2000; Moorthy et al., 2000; Norman and Moerman, 2002). We assessed SNB-1::GFP localization in two $u n c-70$ alleles that encode the $\beta$-spectrin known to be expressed in neurons (Hammarlund et al., 2000; Moorthy et al., 2000). unc-70(e524ts) is a viable semidominant allele in which only $6 \%$ of the animals have any misaccumulation of SNB-1::GFP (Fig. 7b, Table 1). The null allele $u n c-70(s 1502)$ is subviable, and only $25 \%$ of the animals have any misaccumulation of SNB-1::GFP (Hammarlund et al., 2000) (Fig. 7c). Only 33\% of adult animals carrying a null allele in $\beta_{\mathrm{H}^{-}}$-spectrin sma-1, which expresses predominantly in the epithelia of embryos, have misaccumulations in the nose (McKeown et al., 1998). The size of the misaccumulations in all $\beta$-spectrin mutants is smaller than those in $d h c-1$ or $d n c-2$ dynamitin-overexpressing animals (Fig. 7, compare $d, a$ ). For instance, misaccumulations in Sma-1 animals are $\sim 2.5$ times less in size than in dhc- 1 mutants (Table 1 ). The $\beta$-spectrin mutants also differ from the dynein complex mutants in that they show no agerelated increase in the size of misaccumulations and have no hyper-accumulations of SNB-1::GFP in the nerve ring. Surprisingly, these data suggest that spectrin, long proposed as the receptor for dynein- and dynactin-dependent vesicular cargo, is not essential for the transport of neuronal dynein cargo identified here.

\section{Increased severity of some phenotypes with age}

We wanted to determine the consequences of selective retrograde transport defects for the organism. Disruption of certain dynein complex components has been linked to motor neuron disease phenotypes such as ALS (LaMonte et al., 2002; Hafezparast et al., 2003; Puls et al., 2003). A hallmark of such diseases is that phenotypes are progressive, becoming more severe with age and often resulting in death. Interestingly, some phenotypes of $d h c-1$ and $d l i-1$ animals were enhanced with age, and animals died prematurely. The size of the misaccumulations in $d h c-1$ and dli-1 doubled over the course of $5 \mathrm{~d}$ (Fig. 8A), correlating with decreased locomotion. The number of misaccumulations in a neuronal process also increased in older animals. In 1-d-old adult $d h c-1(j s 121)$ and $d l i-1$ (js351) animals, 73\% (27 of 37 ) and $54 \%$ ( 15 of 28 ) of the neuronal processes had only a single misaccumulation. In 5 -d-old adult animals, $\sim 75 \%$ of the neuronal processes had more than one misaccumulation: 15 of 20 and 20 of 26 for $d h c-1(j s 121)$ and $d l i-1(j s 351)$ respectively.
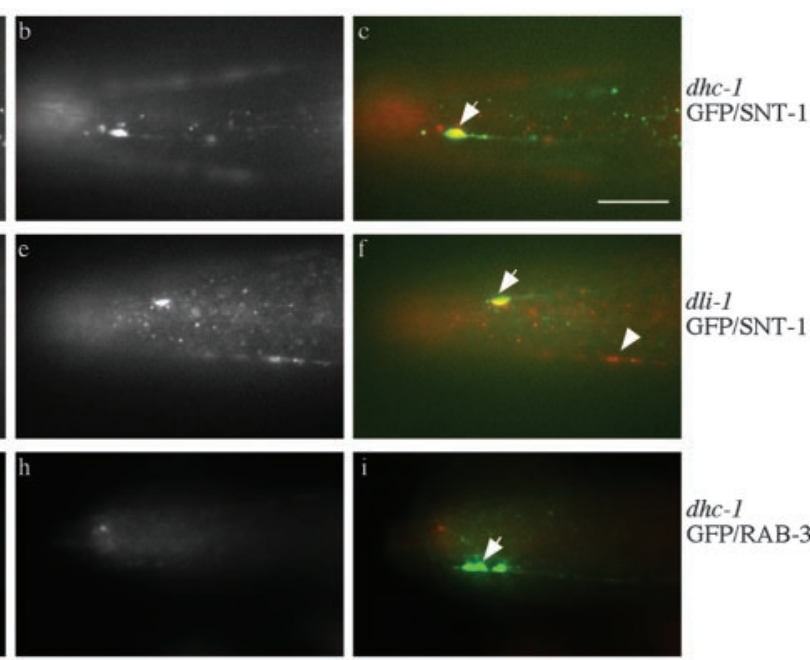

dhc-1 GFP/RAB-3
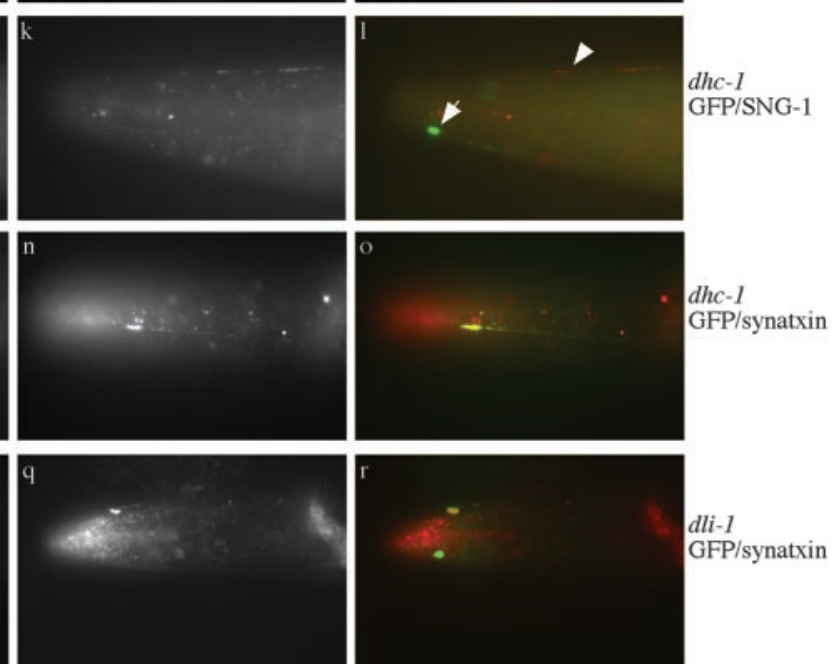

Figure 5. Localization of synaptic proteins in dynein mutants. In all images, arrows point to misaccumulations at the tip of ALM tions in both genotypes contain SNT-1 immunoreactivity. $g-i$, Double labeling of GFP (green, first image of set) and RAB-3 (red ( (

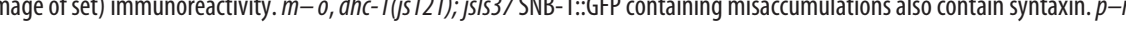
which does not (arrowhead). In general, $\sim 40-50 \%$ of SNB-1::GFP misaccumulations contain syntaxin. The buccal cavity of the animal often shows nonspecific reactivity with many antibodies; this is seen in $b, c, e, f, i, n, 0, q$, and $r$. Scale bar, $10 \mu \mathrm{m}$.

Neuromuscular junction synapses were visualized by SNB-1::GFP expressed in VD and DD motor neurons using the transgenic strain juIs1 (Hallam and Jin, 1998). In 1-d-old adult dhc-1(js121) animals, SNB-1::GFP puncta that marked neuromuscular junction synapses appeared mostly normal, although occasional larger aggregates were observed (Fig. 9b,d). In 5- to 6-d-old adult $d h c-1$ (js121) animals, the SNB-1::GFP pattern was more disorganized compared with age-matched controls. Occasional clumps that may represent cargo accumulations were observed. Furthermore, unusually shaped and widely spaced puncta were observed, consistent with both structural changes in synapse morphology and possible synapse retraction or loss (Fig. 9f, h,j). The number of motor neurons visualized by juIs 1 in 5-d-old $d h c-1$ (js121) adults was not different from that of wild type (data not shown). The $d h c-1$ and $d l i-1$ mutants had locomotory defects even as young adults and moved $\sim 1.3$ times slower than wild type 

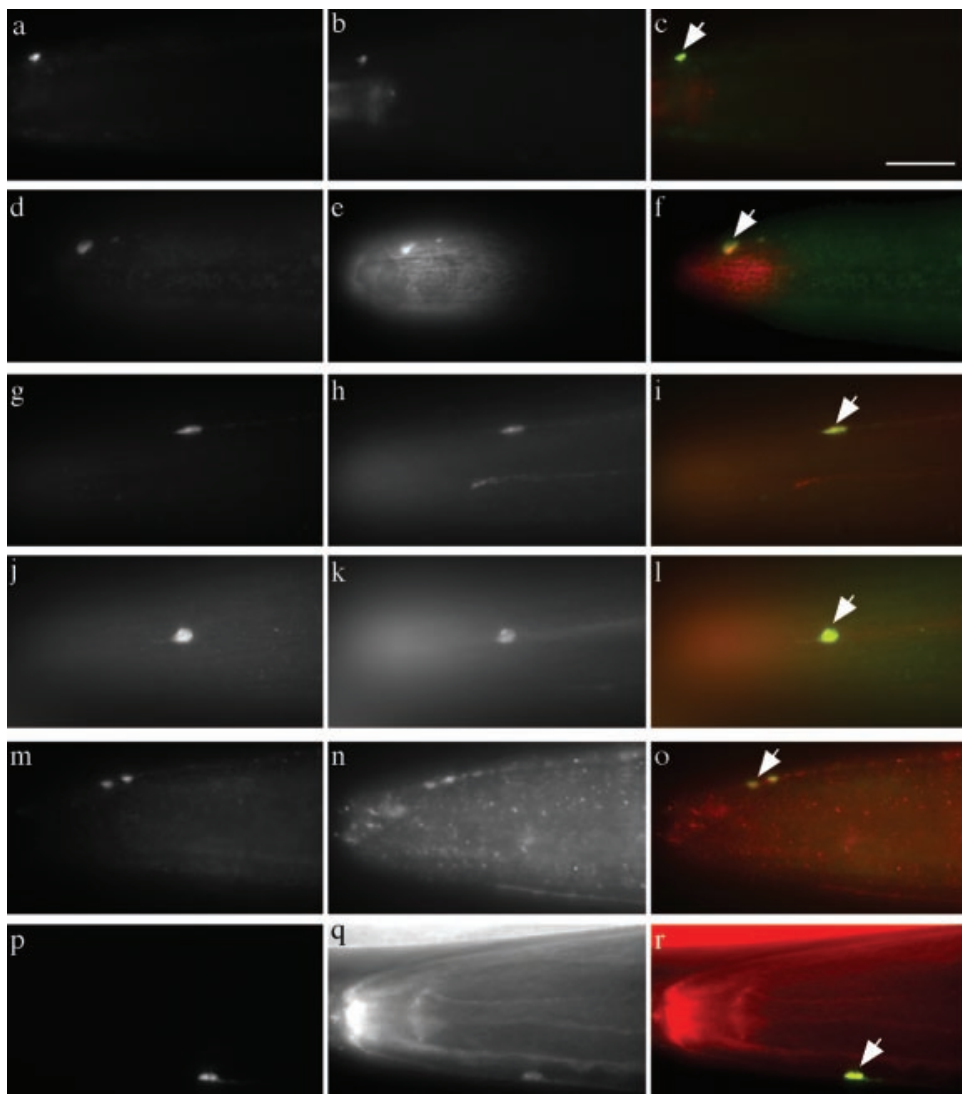

Figure 6. Misaccumulations of protein in $d n c-1$ (or404ts) $\mathrm{p} 150^{\text {Glued }}$ mutant and in overexpression of $d n c-2$ dynamitin in mechanosensory neurons. All arrows point to misaccumulations at the tip of the ALM process in the nose. $a-f$, Double labeling for GFP (green, first image of set) and SNT-1 (red, second image of set) immunoreactivity. $a-c, d n c-1$ (or 404ts). $d-f$, dnc-2 overexpression. $g-I$, Double labeling of GFP (green, first image of set) and UNC-104 (red, second image of set) immunoreactivity. $g-i$, dnc-1(or404ts). j-I, dnc-2 overexpression. $m-r$, Double labeling for GFP (green, first image of set) and UNC-64 syntaxin (red, second image of set) immunoreactivity. $m-0, d n c-1$ (or $404 t 5)$. $p-r$, dnc-2 overexpression. SNB-1::GFP misaccumulations contain SNT-1, UNC-104, and UNC-64 immunoreactivities. The buccal cavity of the animal often shows nonspecific reactivity with many antibodies; this is seen in $f, h, i, k, l, n, 0, q$, and $r$. Scale bar, $10 \mu \mathrm{m}$.
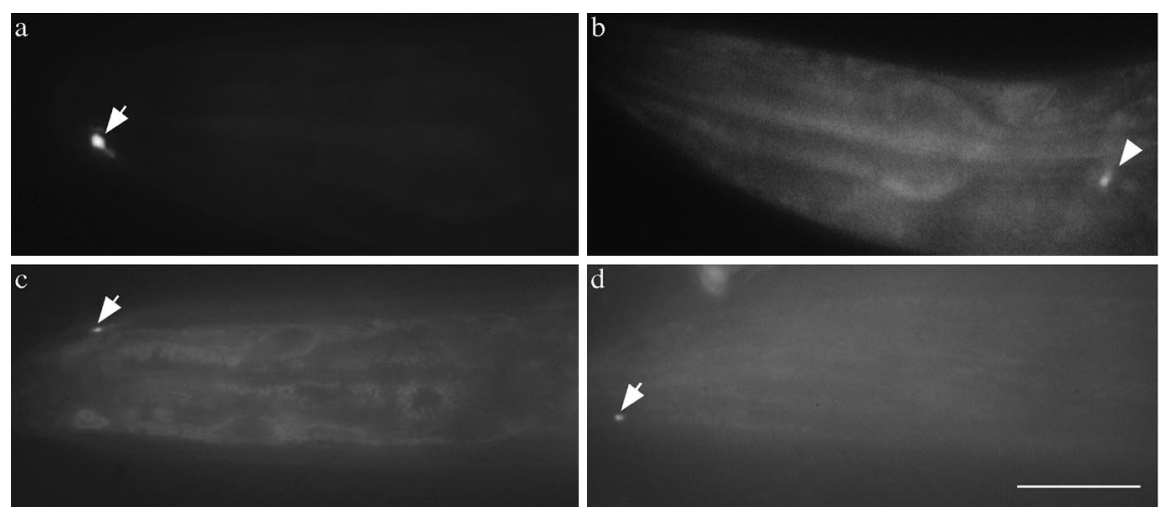

Figure 7. Role of spectrin in localization of SNB-1::GFP in mechanosensory neurons. $a$, Nose of $d$ hc-1 (js 121); js/s37, where the arrow shows an accumulation. b, unc-70(e524); ;s/s37, which does not have any misaccumulation. c, unc-70(s1502); is/s40 with an accumulation (arrow). d, sma-1(ru18); js/s37 animal that has a misaccumulation (arrow). No hyperaccumulations are seen in the nerve ring regions of Unc-70 animals ( $a$, arrowhead; data not shown). Scale bar, $10 \mu \mathrm{m}$.

(Fig. 8B). As wild-type animals age, their velocities decline to approximately half that of young adults. However, the 5-d-old adult $d h c-1$ and $d l i-1$ animals are more severely affected with age and move at velocities fivefold to eightfold slower than 1-d-old mutant animals. As in other animal models for ALS, C. elegans dynein mutants died prematurely. Wild-type adults live dnc-1 GFP/SNT-1

dnc-2

GFP/SNT-1

dnc-1 GFP/syntaxin

$\sim 17.43 \pm 0.98 \mathrm{~d}$ (mean \pm SEM), whereas dhc- 1 and dli-1 adults have average life spans of $9.04 \pm 0.64$ and $10.04 \pm 0.74 \mathrm{~d}$, respectively $\left(p<1 \times 10^{-8}\right)$. It was striking that no mutant animals survived past $15 \mathrm{~d}$, whereas $66 \%$ of the wild-type animals were still alive (Fig. $8 \mathrm{C}$ ). The features of dynein mutants, namely age dependent worsening of locomotion, increase in size and number of misaccumulations, and reduced life span, have all been observed in certain neurodegenerative diseases (Cleveland and Rothstein, 2001).

\section{Discussion}

We have identified the role of the dynein motor in the transport of a subset of synaptic proteins: synaptobrevin, synaptotagmin, UNC-104, and syntaxin. These proteins are likely to be carried in irregularly shaped vesicles and require the light intermediate chain and the dynactin complex for retrograde transport. Interestingly, $\beta$-spectrin mutants do not phenocopy the dynein complex mutants, suggesting alternate ways of linking dynactin-dependent cargo to the dynein motor. Therefore, the dynein-dynactin complex demonstrates cargo specificity and can transport some cargoes without spectrin.

\section{How does the retrograde transport machinery function?}

Vertebrate neurons have complex morphologies. In contrast, $C$. elegans mechanosensory neurons are morphologically simple; nevertheless, these cells have a synaptic branch that extends from the primary process along which synapses are made (Fig. 1d,g) (Chalfie, 1993). Localization of synaptic components to only one branch could be regulated by directed transport. However, the dynein mutants suggest that the transport of synaptobrevin, synaptotagmin, and UNC-104 is not directed but occurs along both the primary process and the synaptic branch. Thus, cargo may be preferentially held or released at the synapse, but the transport machinery is available in all neuronal processes. The misaccumulations at the ends of neuronal processes in the dynein complex mutants indicate a change in the balance of transport, in which retrograde transport is reduced and anterograde transport remains mostly normal.

We favor the hypothesis that the protein misaccumulations arise from changes in axonal transport because the phenotypes arise from mutations in the dynein motor complex and depend on both the anterograde kinesin family motor and microtubules. However, such phenotypes can arise from disruptions in other cellular processes. For instance, changes in endocytosis, altered packaging of pro- 
teins such that they are now targeted to new types of vesicles, the involvement of dynein in turnaround of vesicles, and even altered degradation pathways could all contribute to the aberrant protein accumulations observed.

Cargo selectivity of the dynein complex The dynein complex is the major retrograde motor in axons and may have roles in bulk retrograde transport of many components. Interestingly, only some proteins, synaptobrevin, synaptotagmin, and their anterograde motor UNC-104, always misaccumulate in dynein mutants. We propose that these are dynein cargoes (Figs. $4 a-d, 5 a-f$ ). The lack of effect on other proteins tested could arise from three alternatives. First, some proteins are known to be degraded at the synapse (Klimaschewski, 2003), making them unavailable for retrograde transport. For example, in vertebrate axons, only $30 \%$ of the anterogradely transported amphiphysin and synaptojanin, and none of the Rab3a was retrogradely transported (Li et al., 1995, 1997). Second, other motors could retrogradely transport some proteins. Good candidates for alternate motors are the C-terminal kinesins, which, thus far, have all been microtubule minus end-directed and hence well suited to carrying cargo back to the cell body (Henikoff et al., 1996). There are four C-terminal kinesins in C. elegans, but their role in axonal transport remains unknown (Siddiqui, 2002). The occasional misaccumulation of the plasma membrane transmembrane protein syntaxin indirectly supports the idea that other motors could be involved. Third, adequate levels of transport by the remaining dynein complex may allow some retrograde transport in these mutants.

It is unknown whether the transmembrane proteins synaptobrevin, synaptotagmin, and syntaxin are full-length and present on the same transport vesicle. Near the tips of the mechanosensory neuronal process, numerous irregular tubovesicular profiles are observed, which are likely to contain the misaccumulating proteins seen by light microscopy (Fig. $2 f, g$ ). If a single vesicle contains all three transmembrane proteins, it must arise by complex processes because syntaxin is primarily a plasma membrane protein, whereas synaptobrevin and synaptotagmin are synaptic vesicle proteins.

\section{Cargo recognition}

This study highlights differential cargo recognition by the large multisubunit dynein-dynactin complex. We show that a partial loss-of-function mutation in $d n c-1$ p150 ${ }^{\text {Glued }}$ and overexpression of $d n c-2$ dynamitin (Skop and White, 1998), which disassembles the dynactin complex (Echeverri et al., 1996), have phenotypes identical to $d h c-1$ and $d l i-1$. Thus, synaptobrevin-, synaptotagmin-, and UNC-104-containing vesicles require the dynactin complex as well as the dynein motor complex for trans-
B

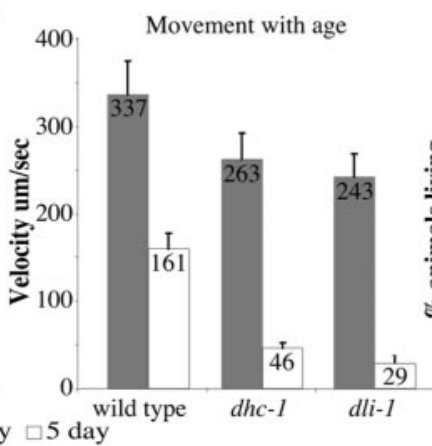

C

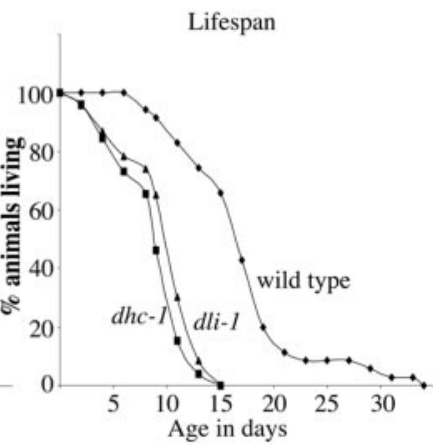

Figure 8. Age dependent effects of the mutants. A, Size of accumulations of 1- and 5-d-old adult dhc-1(js 121 ); is/s37 and $b$ and $c$, for how numbers of misaccumulations can vary in a single neuronal process. The increase in size of misaccumulations is 2.5 times as the animal ages $(n=20)$. The 1 and $5 \mathrm{~d}$ values are significantly different for both genotypes $(p<5 \times 10$ js/s37, dhc-1(js121); js/s37, and dli-1(js351); js/s37 adult animals of $1 \mathrm{~d}(n=21)$ and $5 \mathrm{~d}(n \geq 17)$. Velocity measurements of both as velocity \pm SD. C, Life span curves. Animals were collected as late L4 larvae ( $0 \mathrm{~d})$ and followed every other day until they died. Wild-type jsls37, dhc-1(js121); jsls37, and dli-1(js351); js/s37 animals have $n=35,26$, and 23, respectively.
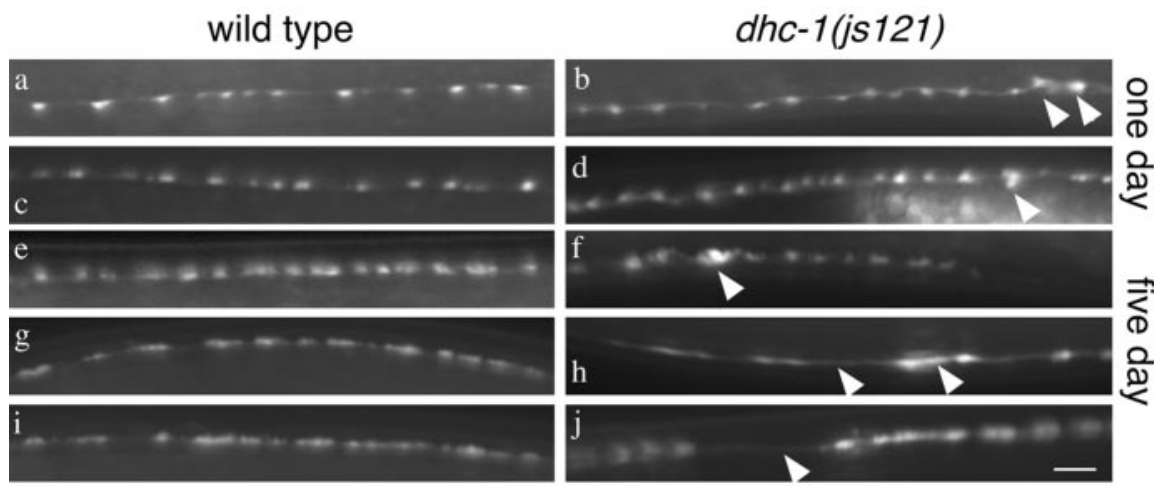

Figure 9. Neuromuscular junctions as observed by SNB-1::GFP expression in VD and DD motor neurons. $a-d, 0$ ne-day-old dhc-1(js121); juls1 ( $f, h, j)$. Arrowheads point to clumps of SNB-1::GFP, gaps in SNB-1::GFP puncta and other irregularities in SNB-1::GFP localization in mutant animals. Scale bar, $5 \mu \mathrm{m}$.

port. Interaction of the vesicular cargo with dynactin is thought to require the hetrotetramer spectrin, whose subunit $\beta$-spectrin binds to the ARP1 filament of dynactin (Holleran et al., 1996, 2001; Muresan et al., 2001). Interestingly, only 35\% of loss-offunction mutant animals in both C. elegans $\beta$-spectrins have misaccumulations of SNB-1::GFP at the tips of neuronal processes (Table 1) and no hyperaccumulations near the nerve ring. The misaccumulations in the $\beta$-spectrin null mutants are also significantly smaller than those seen in the partial loss-of-function dhc-1 mutants. C. elegans has two types of spectrin tetramers, $(\alpha \beta)_{2}$ and $\left(\alpha \beta_{\mathrm{H}}\right)_{2}$, formed by SPC-1, UNC-70, and SMA-1 (Bennett and Baines, 2001). Of these, the UNC-70 $\beta$-spectrin is enriched in neurons, whereas neuronal roles of SMA- $1 \beta_{\mathrm{H}^{-}}$spectrin and SPC- $1 \alpha$-spectrin have not been described (Hammarlund et al., 2000; Moorthy et al., 2000). We were unable to directly address whether either $\beta$-spectrin gene could compensate for the other or to assess the phenotype of an $\alpha$-spectrin mutant because animals lacking both $\beta$-spectrins or $\alpha$-spectrin die as embryos (Norman and Moerman, 2002). However, our data suggest that vesicles containing synaptobrevin are not critically dependent on spectrin for cargo recognition. In support of this, the UNC-70 
protein does not misaccumulate with SNB-1::GFP in the dynein complex mutants (data not shown). The misaccumulation defects in SMA-1 may arise from dynein-independent effects. Spectrin probably plays important roles in transport of other dyneinand dynactin-dependent vesicles that we have not assayed.

Although the spectrin-dynactin-dynein link is thought to be important in cargo recognition and motility, recruitment of the complex to a transport vesicle can occur by other means. It is known that Rab6 GTPase, Bicaudal-D, and Rab7 effector Rab7 interacting lysosomal protein can all recruit dynein-dynactin complexes to Golgi-derived membranes and lysosomes (Jordens et al., 2001; Matanis et al., 2002; Short et al., 2002). The dynactin complex could function independent of cargo recognition and instead could modulate the processivity of the dynein motor (Waterman-Storer et al., 1995; King and Schroer, 2000). Because the genetic screen that isolated the dynein mutants was not saturating, other genes that aid in cargo recognition may yet be recovered by isolating mutants with similar phenotypes.

Are there parallels to neurodegenerative diseases such as ALS? There are two lines of evidence suggesting that altered transport contributes to ALS pathologies. First, slowed transport is an early and presymptomatic event in a mouse model of ALS (Williamson and Cleveland, 1999). Second, mutations in the human and mouse dynein-dynactin complex mimic phenotypes seen in ALS (Hafezparast et al., 2003; Puls et al., 2003). We show that $C$. elegans dynein complex mutants have interesting parallels to ALS disease models. Notable among these are the increase in size and frequency of misaccumulations found in neuronal processes. Similar increases in size and number of protein aggregates with age have been observed in ALS mouse models (Cleveland and Rothstein, 2001; LaMonte et al., 2002). Proteins such as neurofilaments, ubiquitin, superoxide dismutase, and $\beta$-tubulin are known to accumulate in affected neurons (Williamson and Cleveland, 1999; Cleveland and Rothstein, 2001; LaMonte et al., 2002; Hafezparast et al., 2003). Of these, we were able to assess only $\beta$-tubulin misaccumulation; in $d h c-1, d l i-1, d n c-1$, and $d n c-2$ dynamitin-overexpressing animals, $30-40 \%$ of synaptobrevin misaccumulations also contain $\beta$-tubulin (data not shown).

Other progressive phenotypes seen in the C. elegans dynein complex mutants include reduced locomotion with age and a $42 \%$ reduced life span. The locomotory deficits may result from the synaptic changes observed at the neuromuscular junctions (Fig. 9). However, locomotory and life span phenotypes could have significant contributions from the non-neuronal roles of dynein. The contribution of reduced dynein function exclusively in neurons to the observed progressive $C$. elegans phenotypes remains to be determined. This question is incompletely understood even in the mouse models of ALS, especially because motor neuron degeneration is not neuronal cell type-autonomous (Pramatarova et al., 2001; Lino et al., 2002; Clement et al., 2003). Whether motor neuron degeneration similar to that seen in ALS occurs in C. elegans dynein mutants is unclear.

Lesions in retrograde transport proteins have been shown to be an important component of motor neuron diseases in both mice and humans (LaMonte et al., 2002; Hafezparast et al., 2003; Puls et al., 2003). How could alterations in selective retrograde transport, as seen in this model system, lead to neurodegeneration? An important role of retrograde transport may be to clear unwanted components from synaptic regions. The misaccumulation of specific proteins or even the physical occlusion of transport pathways could have implications for disease. C. elegans dy- nein mutants provide a simple genetic system to help address these issues.

\section{References}

Bennett V, Baines AJ (2001) Spectrin and ankyrin-based pathways: metazoan inventions for integrating cells into tissues. Physiol Rev 81:1353-1392.

Bowman AB, Patel-King RS, Benashski SE, McCaffery JM, Goldstein LS, King SM (1999) Drosophila roadblock and Chlamydomonas LC7: a conserved family of dynein-associated proteins involved in axonal transport, flagellar motility, and mitosis. J Cell Biol 146:165-180.

Chalfie M (1993) Touch receptor development and function in Caenorhabditis elegans. J Neurobiol 24:1433-1441.

Chalfie M, Thomson JN (1982) Structural and functional diversity in the neuronal microtubules of Caenorhabditis elegans. J Cell Biol 93:15-23.

Clement AM, Nguyen MD, Roberts EA, Garcia ML, Boillee S, Rule M, McMahon AP, Doucette W, Siwek D, Ferrante RJ, Brown Jr RH, Julien JP, Goldstein LS, Cleveland DW (2003) Wild-type nonneuronal cells extend survival of SOD1 mutant motor neurons in ALS mice. Science 302:113-117.

Cleveland DW, Rothstein JD (2001) From Charcot to Lou Gehrig: deciphering selective motor neuron death in ALS. Nat Rev Neurosci 2:806-819.

Echeverri CJ, Paschal BM, Vaughan KT, Vallee RB (1996) Molecular characterization of the $50-\mathrm{kD}$ subunit of dynactin reveals function for the complex in chromosome alignment and spindle organization during mitosis. J Cell Biol 132:617-633.

Fukushige T, Siddiqui ZK, Chou M, Culotti JG, Gogonea CB, Siddiqui SS, Hamelin M (1999) MEC-12, an (alpha)-tubulin required for touch sensitivity in C. elegans. J Cell Sci 112:395-403.

Gindhart Jr JG, Desai CJ, Beushausen S, Zinn K, Goldstein LS (1998) Kinesin light chains are essential for axonal transport in Drosophila. J Cell Biol 141:443-454

Gindhart JG, Chen J, Faulkner M, Gandhi R, Doerner K, Wisniewski T, Nandlestadt A (2003) The kinesin-associated protein UNC-76 is required for axonal transport in the Drosophila nervous system. Mol Biol Cell 14:3356-3365.

Goldstein LS, Yang Z (2000) Microtubule-based transport systems in neurons: the roles of kinesins and dyneins. Annu Rev Neurosci 23:39-71.

Gonczy P, Pichler S, Kirkham M, Hyman AA (1999) Cytoplasmic dynein is required for distinct aspects of MTOC positioning, including centrosome separation, in the one cell stage Caenorhabditis elegans embryo. J Cell Biol 147:135-150.

Hafezparast M, Klocke R, Ruhrberg C, Marquardt A, Ahmad-Annuar A, Bowen S, Lalli G, Witherden AS, Hummerich H, Nicholson S, Morgan PJ, Oozageer R, Priestley JV, Averill S, King VR, Ball S, Peters J, Toda T, Yamamoto A, Hiraoka Y, Augustin M, Korthaus D, Wattler S, Wabnitz P, Dickneite C, Lampel S, Boehme F, Peraus G, Popp A, Rudelius M, Schlegel J, Fuchs H, de Angelis MH, Schiavo G, Shima DT, Russ AP, Stumm G, Martin JE, Fisher EM (2003) Mutations in dynein link motor neuron degeneration to defects in retrograde transport. Science 300:808-812.

Hall DH, Hedgecock EM (1991) Kinesin-related gene unc-104 is required for axonal transport of synaptic vesicles in C. elegans. Cell 65:837-847.

Hallam SJ, Jin Y (1998) lin-14 regulates the timing of synaptic remodelling in Caenorhabditis elegans. Nature 395:78-82.

Hamill DR, Severson AF, Carter JC, Bowerman B (2002) Centrosome maturation and mitotic spindle assembly in C. elegans require SPD-5, a protein with multiple coiled-coil domains. Dev Cell 3:673-684.

Hammarlund M, Davis WS, Jorgensen EM (2000) Mutations in betaspectrin disrupt axon outgrowth and sarcomere structure. J Cell Biol 149:931-942.

Harada A, Takei Y, Kanai Y, Tanaka Y, Nonaka S, Hirokawa N (1998) Golgi vesiculation and lysosome dispersion in cells lacking cytoplasmic dynein. J Cell Biol 141:51-59.

Henikoff S, Endow SA, Greene EA (1996) Connecting protein family resources using the proWeb network. Trends Biochem Sci 21:444-445.

Hirokawa N, Sato-Yoshitake R, Yoshida T, Kawashima T (1990) Brain dynein (MAP1C) localizes on both anterogradely and retrogradely transported membranous organelles in vivo. J Cell Biol 111:1027-1037.

Holleran EA, Tokito MK, Karki S, Holzbaur EL (1996) Centractin (ARP1) associates with spectrin revealing a potential mechanism to link dynactin to intracellular organelles. J Cell Biol 135:1815-1829. 
Holleran EA, Ligon LA, Tokito M, Stankewich MC, Morrow JS, Holzbaur EL (2001) beta III spectrin binds to the Arp1 subunit of dynactin. J Biol Chem 276:36598-36605.

Hurd DD, Saxton WM (1996) Kinesin mutations cause motor neuron disease phenotypes by disrupting fast axonal transport in Drosophila. Genetics 144:1075-1085.

Jordens I, Fernandez-Borja M, Marsman M, Dusseljee S, Janssen L, Calafat J, Janssen H, Wubbolts R, Neefjes J (2001) The Rab7 effector protein RILP controls lysosomal transport by inducing the recruitment of dyneindynactin motors. Curr Biol 11:1680-1685.

King SJ, Schroer TA (2000) Dynactin increases the processivity of the cytoplasmic dynein motor. Nat Cell Biol 2:20-24.

King SJ, Bonilla M, Rodgers ME, Schroer TA (2002) Subunit organization in cytoplasmic dynein subcomplexes. Protein Sci 11:1239-1250.

King SM (2000) The dynein microtubule motor. Biochim Biophys Acta 1496:60-75.

Klimaschewski L (2003) Ubiquitin-dependent proteolysis in neurons. News Physiol Sci 18:29-33.

Koushika SP, Richmond JE, Hadwiger G, Weimer RM, Jorgensen EM, Nonet ML (2001) A post-docking role for active zone protein Rim. Nat Neurosci 4:997-1005.

Labrousse AM, Shurland DL, van der Bliek AM (1998) Contribution of the GTPase domain to the subcellular localization of dynamin in the nematode Caenorhabditis elegans. Mol Biol Cell 9:3227-3239.

LaMonte BH, Wallace KE, Holloway BA, Shelly SS, Ascano J, Tokito M, Van Winkle T, Howland DS, Holzbaur EL (2002) Disruption of dynein/dynactin inhibits axonal transport in motor neurons causing late-onset progressive degeneration. Neuron 34:715-727.

Li JY, Jahn R, Dahlstrom A (1995) Rab3a, a small GTP-binding protein, undergoes fast anterograde transport but not retrograde transport in neurons. Eur J Cell Biol 67:297-307.

Li JY, De Camilli P, Dahlstrom A (1997) Intraneuronal trafficking and distribution of amphiphysin and synaptojanin in the rat peripheral nervous system and the spinal cord. Eur J Neurosci 9:1864-1874.

Lino MM, Schneider C, Caroni P (2002) Accumulation of SOD1 mutants in postnatal motoneurons does not cause motoneuron pathology or motoneuron disease. J Neurosci 22:4825-4832.

Martin M, Iyadurai SJ, Gassman A, Gindhart Jr JG, Hays TS, Saxton WM (1999) Cytoplasmic dynein, the dynactin complex, and kinesin are interdependent and essential for fast axonal transport. Mol Biol Cell 10:3717-3728.

Matanis T, Akhmanova A, Wulf P, Del Nery E, Weide T, Stepanova T, Galjart N, Grosveld F, Goud B, De Zeeuw CI, Barnekow A, Hoogenraad CC (2002) Bicaudal-D regulates COPI-independent Golgi-ER transport by recruiting the dynein-dynactin motor complex. Nat Cell Biol 4:986-992.

McKeown C, Praitis V, Austin J (1998) sma-1 encodes a betaH-spectrin homolog required for Caenorhabditis elegans morphogenesis. Development 125:2087-2098.

Moorthy S, Chen L, Bennett V (2000) Caenorhabditis elegans beta-G spectrin is dispensable for establishment of epithelial polarity, but essential for muscular and neuronal function. J Cell Biol 149:915-930.

Muresan V, Stankewich MC, Steffen W, Morrow JS, Holzbaur EL, Schnapp BJ (2001) Dynactin-dependent, dynein-driven vesicle transport in the absence of membrane proteins: a role for spectrin and acidic phospholipids. Mol Cell 7:173-183.

Nonet ML (1999) Visualization of presynaptic terminal specializations in live C. elegans using synaptic vesicle protein-GFP fusions. J Neurosci Methods 89:33-40.

Nonet ML, Grundahl K, Meyer BJ, Rand JB (1993) Synaptic function is impaired but not eliminated in C. elegans mutants lacking synaptotagmin. Cell 73:1291-1305.

Nonet ML, Staunton JE, Kilgard MP, Fergestad T, Hartwieg E, Horvitz HR, Jorgensen EM, Meyer BJ (1997) Caenorhabditis elegans rab-3 mutant synapses exhibit impaired function and are partially depleted of vesicles. J Neurosci 17:8061-8073.

Nonet ML, Saifee O, Zhao H, Rand JB, Wei L (1998) Synaptic transmission deficits in Caenorhabditis elegans synaptobrevin mutants. J Neurosci 18:70-80.

Norman KR, Moerman DG (2002) Alpha spectrin is essential for morphogenesis and body wall muscle formation in Caenorhabditis elegans. J Cell Biol 157:665-677.
Otsuka AJ, Jeyaprakash A, Garcia-Anoveros J, Tang LZ, Fisk G, Hartshorne T, Franco R, Born T (1991) The C. elegans unc-104 gene encodes a putative kinesin heavy chain-like protein. Neuron 6:113-122.

Pramatarova A, Laganiere J, Roussel J, Brisebois K, Rouleau GA (2001) Neuron-specific expression of mutant superoxide dismutase 1 in transgenic mice does not lead to motor impairment. J Neurosci 21:3369-3374.

Puls I, Jonnakuty C, LaMonte BH, Holzbaur EL, Tokito M, Mann E, Floeter MK, Bidus K, Drayna D, Oh SJ, Brown Jr RH Jr, Ludlow CL, Fischbeck KH (2003) Mutant dynactin in motor neuron disease. Nat Genet 33:455-456.

Saifee O, Wei L, Nonet ML (1998) The Caenorhabditis elegans unc-64 locus encodes a syntaxin that interacts genetically with synaptobrevin. Mol Biol Cell 9:1235-1252.

Savage C, Hamelin M, Culotti JG, Coulson A, Albertson DG, Chalfie M (1989) mec-7 is a beta-tubulin gene required for the production of $15-$ protofilament microtubules in Caenorhabditis elegans. Genes Dev 3:870-881.

Savage C, Xue Y, Mitani S, Hall D, Zakhary R, Chalfie M (1994) Mutations in the Caenorhabditis elegans beta-tubulin gene mec-7: effects on microtubule assembly and stability and on tubulin autoregulation. J Cell Sci 107:2165-2175.

Schaefer AM, Hadwiger GD, Nonet ML (2000) rpm-1, a conserved neuronal gene that regulates targeting and synaptogenesis in C. elegans. Neuron 26:345-356.

Schnapp BJ, Reese TS (1989) Dynein is the motor for retrograde axonal transport of organelles. Proc Natl Acad Sci USA 86:1548-1552.

Short B, Preisinger C, Schaletzky J, Kopajtich R, Barr FA (2002) The Rab6 GTPase regulates recruitment of the dynactin complex to Golgi membranes. Curr Biol 12:1792-1795.

Siddiqui SS (2002) Metazoan motor models: kinesin superfamily in C. elegans. Traffic 3:20-28.

Skop AR, White JG (1998) The dynactin complex is required for cleavage plane specification in early Caenorhabditis elegans embryos. Curr Biol 8:1110-1116.

Staunton J, Ganetzky B, Nonet ML (2001) Rabphilin potentiates soluble $\mathrm{N}$-ethylmaleimide-sensitive factor attachment protein receptor function independently of rab3. J Neurosci 21:9255-9264.

Steffen W, Langford GM, Weiss DG, Kuznetsov SA (1997) Inhibition of microtubule-dependent, minus-end directed transport of axoplasmic organelles by an antibody specific for the intermediate chain of dynein. Biol Bull 193:221-222.

Sulston J, Hodgkin J (1988) Methods. In: The nematode Caenorhabditis elegans (Wood WB, ed), pp 587-606. Cold Spring Harbor, NY: Cold Spring Harbor Laboratory.

Susalka SJ, Hancock WO, Pfister KK (2000) Distinct cytoplasmic dynein complexes are transported by different mechanisms in axons. Biochim Biophys Acta 1496:76-88.

Waterman-Storer CM, Karki S, Holzbaur EL (1995) The p150Glued component of the dynactin complex binds to both microtubules and the actin-related protein centractin (Arp-1). Proc Natl Acad Sci USA 92:1634-1638.

Waterman-Storer CM, Karki SB, Kuznetsov SA, Tabb JS, Weiss DG, Langford GM, Holzbaur EL (1997) The interaction between cytoplasmic dynein and dynactin is required for fast axonal transport. Proc Natl Acad Sci USA 94:12180-12185.

Wicks SR, Yeh RT, Gish WR, Waterston RH, Plasterk RH (2001) Rapid gene mapping in Caenorhabditis elegans using a high density polymorphism map. Nat Genet 28:160-164.

Williams BD, Schrank B, Huynh C, Shownkeen R, Waterston RH (1992) A genetic mapping system in Caenorhabditis elegans based on polymorphic sequence-tagged sites. Genetics 131:609-624.

Williamson TL, Cleveland DW (1999) Slowing of axonal transport is a very early event in the toxicity of ALS-linked SOD1 mutants to motor neurons. Nat Neurosci 2:50-56.

Yoder JH, Han M (2001) Cytoplasmic dynein light intermediate chain is required for discrete aspects of mitosis in Caenorhabditis elegans. Mol Biol Cell 12:2921-2933.

Zhao H, Nonet ML (2000) A retrograde signal is involved in activitydependent remodeling at a C. elegans neuromuscular junction. Development 127:1253-1266.

Zhao H, Nonet ML (2001) A conserved mechanism of synaptogyrin localization. Mol Biol Cell 12:2275-2289. 